\title{
The Role of Cardiac MRI in the Diagnosis and Management of Loeffler's Endocarditis: A Case Report with Clinical and Pathologic Correlation
}

\author{
K. Chad Hilty ${ }^{\mathrm{a}}$, James A. Koonce ${ }^{\mathrm{b}}$, Roger W. Stone ${ }^{\mathrm{c}}$, Luis Ramos-Duran ${ }^{\mathrm{b}}$, Ashley L. Pyle ${ }^{\mathrm{d}}$, Nicholas \\ I. Batalis ${ }^{\mathrm{c}}$, Andrew D. Hardie ${ }^{\#, \mathrm{~b}}$ and Marian H. Taylor*,\#,a
}

\author{
${ }^{a}$ The Division of Cardiology (Department of Medicine), the ${ }^{b}$ Department of Radiology and Radiological Science, the \\ ${ }^{c}$ Department of Pathology and Laboratory Medicine, and the ${ }^{d}$ Division of General Internal Medicine and Geriatrics \\ (Department of Medicine), Medical University of South Carolina, Charleston, SC 29425, USA
}

\begin{abstract}
Cardiac manifestations of the hypereosinophilic syndrome (HES), first described by Loeffler in 1936, are rare and difficult to image. We report a fatal case of Loeffler's endocarditis in a 71 year-old female with a history of asthma. An initial transthoracic echocardiogram demonstrated normal wall motion and a suggestion of left ventricular apical thrombus. However, using cardiac MRI with ultra fast inversion-recovery (IR) delayed enhancement, gradient recalled echo (GRE) grid-tagging and cine steady-state free precession (SSFP) sequences, we were able to clearly demonstrate endocardial fibrosis, tissue inflammation, subendocardial contractile dysfunction, and mural thrombus that correlate with histopathologic findings at autopsy. We believe cardiac MRI is more useful than transthoracic echocardiography in the diagnosis and management of HES and ultimately may obviate the need for cardiac biopsy to confirm the diagnosis.
\end{abstract}

Keywords: Loeffler's, hypereosinophilic syndrome, MRI, endocarditis.

\section{BACKGROUND}

The Hypereosinophilic syndrome was first described by William Loeffler, a professor of medicine at the University of Zurich, Switzerland, in 1936 [1]. The title of his publication was, "Die Fluechtigen Lungeninfiltrate mit Eosinophilie" (Fleeting Lung Infiltrates with Eosinophilia); a description of a characteristic physical finding of this disorder. It wasn't until 1968, when Hardy and Anderson published a paper entitled the "Hypereosinophilic Syndromes", that Loeffler's endocarditis was recognized as but one manifestation of a larger multi-system disorder [2]. Shortly thereafter, Parrillo, et al., published a prospective study of 26 patients with HES that provided a clinical link between HES and Loeffler's endocarditis, demonstrating that approximately 40$50 \%$ of patients with HES have cardiac involvement [3]. In 1982, Anthony S. Fauci and colleagues at the NIH produced a consensus statement describing the 3 essential criteria for the diagnosis of the hypereosinophic syndrome, namely: peripheral blood eosinophilia of more than 1500 eosinophils/mL for at least 6 months duration, characteristic end organ damage, and no evidence of eosinophil clonality [4]. In this case report, we describe a patient with hypereosinophilic syndrome and Loeffler's endocarditis, for which cardiac MRI yielded significant additional diagnostic and prognostic information (not found on transthoracic echocardiography) that was shown to correlate highly with clinical and histopathologic findings.

\section{Case Report}

A 71 year old Caucasian female was admitted to the cardiology service at the Medical University of South Carolina

\footnotetext{
*Address correspondence to this author at the Medical University of South Carolina, Cardiology Division, 25 Courtenay Drive, ART 7058, Box 592, Charleston, SC 29425, USA; Tel: (843) 876-4763; Fax: (843) 876-4809;

E-mail: taylormh@musc.edu

"Senior co-authors of this manuscript.
}

(MUSC) for the evaluation of progressive dyspnea and heart failure. Her past medical history was remarkable for asthma with eosinophilia (first diagnosed at age 52), three episodes of pneumonia, gastroesophageal reflux disease, and diverticulosis. She was a lifelong non-smoker and denied any known respiratory exposures. After her initial diagnosis of asthma, she had been treated intermittently with oral prednisone in addition to inhaled bronchodilators and corticosteroids. On admission, laboratory studies were significant for a white blood cell count of $13 \mathrm{k} / \mathrm{mm}^{3}$ with $24 \%$ eosinophils and an absolute eosinophil count of $3.3 \mathrm{k} / \mathrm{mm}^{3}$ (reference 0 $0.5)$. An initial computed tomography (CT) scan of the chest showed trace pleural effusions and predominant lower lobe alveolar infiltrates. Pulmonary function tests (PFTs) demonstrated mixed restrictive and obstructive lung disease. A transthoracic echocardiogram (TTE) demonstrated preserved left ventricular systolic function with right atrial and right ventricular dilation and hypokinesis, a dilated coronary sinus, elevated right ventricular systolic pressure, and a laminar echodensity along the apex of the left ventricle, consistent with possible left ventricular apical thrombus (Fig. 1). Cardiac magnetic resonance imaging (MRI) was obtained at this point to further characterize the left ventricular findings on echocardiogram (Fig. 2). It revealed an extensive amount of non-enhancing proteinaceous debris, obliterating the medial and apical segments of the left ventricular lumen as well as apical and septal hypokinesis with intense linear subendocardial hyperenhancement along the same anatomical distribution noted on the delayed-enhancement gadolinium viability sequences. The findings were consistent with early necrotic stage endomyocardial fibrosis with apical and septal involvement of the left ventricle and mild to moderate segmental systolic dysfunction, suggesting a diagnosis of Loeffler's endocarditis with restrictive ventricular function. Left and right heart catheterization (with biopsy), demonstrated normal coronary arteries, elevated left and right ventricular 


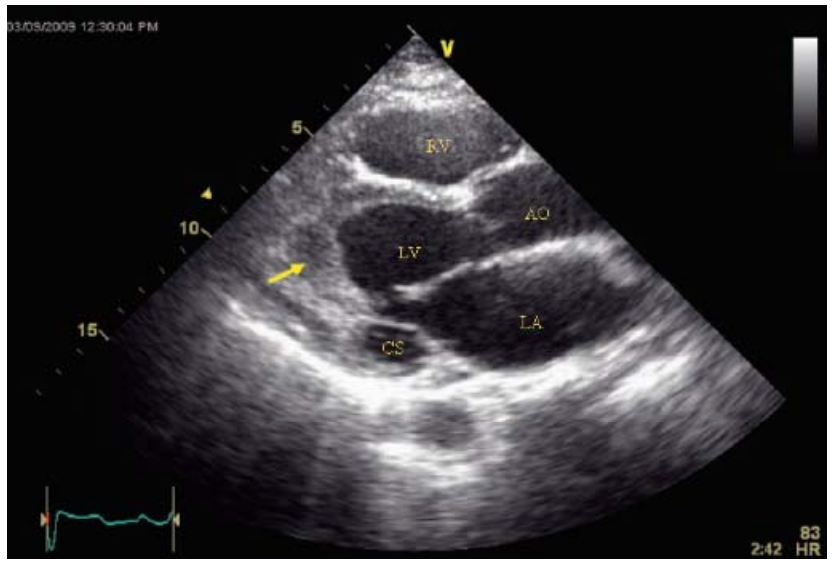

Fig. (1). Transthoracic echocardiogram in the parasternal long-axis view, demonstrating an enlarged left atrium and right ventricle, a dilated coronary sinus, and a linear echodensity in the apex of the left ventricle which was concerning for thrombus (arrow). Labels:, AO - ascending aorta, CS - coronary sinus, LA - left atrium, LV left ventricle, and RV - right ventricle.

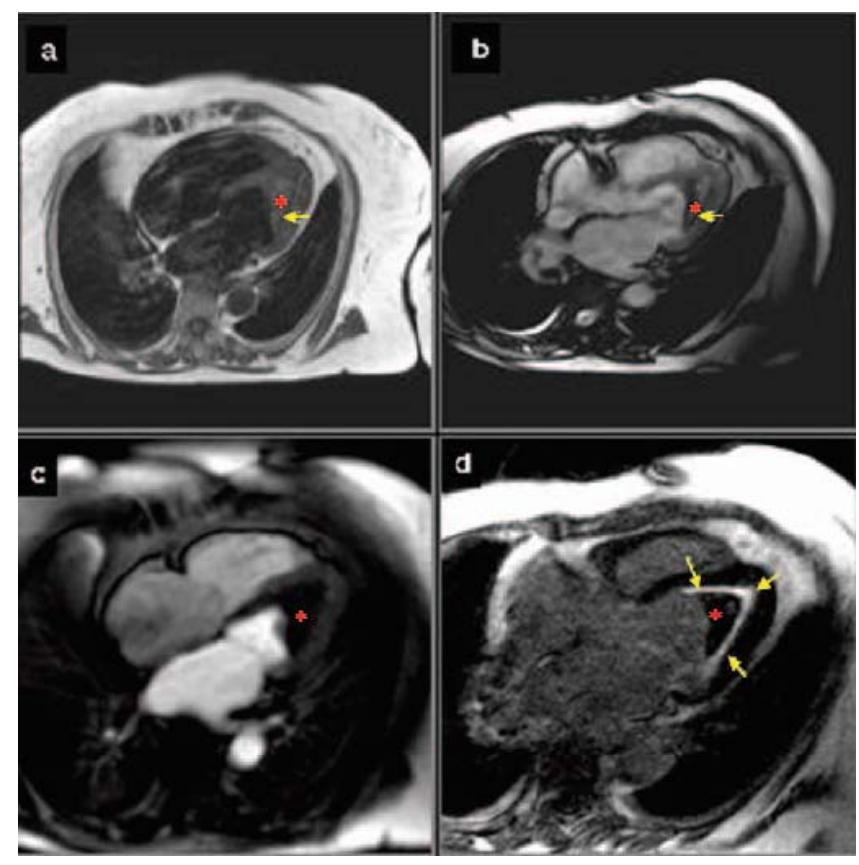

Fig. (2). Cardiac MRI with 4 horizontal long-axis (4 chamber) views: (a) ultra fast double inversion recovery; (b) steady-state free precession; (c) first-pass perfusion; and (d) delayed enhancement. A red asterisk denotes an endocavitary filling defect in the apex of the left ventricle. Image (d) demonstrates lack of enhancement within the filling defect, consistent with thrombus. Image (d) also demonstrates a thin area of delayed enhancement (yellow arrows) distributed along the endocardium of the left ventricle, subtending the distribution of the thrombus. This region of endocardial enhancement (a) is markedly hypointense (yellow arrow), indicating dense subendocardial fibrosis. Note that the remainder of the myocardium deep to the subendocardial fibrosis shows normal signal intensity and perfusion.

end-diastolic pressures, decreased cardiac output and evidence for restrictive physiology. Following the catheterization, the patient became hypoxic and hypotensive and was admitted to the cardiac intensive care unit. Anticoagulation was continued and she was started on high dose intravenous methylprednisolone in addition to standard treatment for heart failure. A hematology consult was obtained, suggesting a bone-marrow biopsy and additional laboratory studies to further characterize the patient's HES. Unfortunately, the patient acutely decompensated 3 days after admission, despite maximal medical therapy. After a brief resuscitation, a bedside echocardiogram revealed new moderate to severe mitral regurgitation and moderate tricuspid regurgitation. Consideration was given to possible emergent surgical intervention, however due to patient and family wishes, supportive measures were withdrawn and the patient died shortly thereafter.

\section{Pathology}

At autopsy, the patient was found to have a $4 \mathrm{~cm}$ layered, dark red to tan mural thrombus in the lumen of the left ventricle involving the chordae tendineae of the mitral valve. This thrombus was overlying a fibrous, thickened left ventricular endocardium (Fig. 3). Microscopically, the left ventricular endocardium demonstrated marked fibrous thickening with associated areas of inflammation consisting predominantly of eosinophils within the fibrous tissue and immediate subendocardium. The thrombus within the left ventricle was composed of fibrin, platelets and red blood cells with marked organization and areas of neovascularization. Sections of the left ventricular myocardium showed scattered inflammation consisting almost exclusively of eosinophils with focal areas of associated myocyte necrosis and myocardial fibrosis (Fig. 4). The patient was also found to have evidence of hypertensive cardiovascular disease which included cardiomegaly (510 grams), mild left ventricular hypertrophy (wall thickness $=1.5 \mathrm{~cm}$ ), granular, pitted, cystic kidneys, and moderate intimal hyperplasia of the renal arterioles. Additionally, the right pulmonary artery contained occlusive thromboemboli extending into the peripheral vasculature. There was patchy consolidation of the right lung as well as multifocal firm, wedge-shaped, red-tan infarcts. Large mucous plugs and mild edema were present bilaterally. Sections from the right and left lungs showed thickened basement membranes with muscular hypertrophy, chronic interstitial inflammation with scattered eosinophils, mild fibrosis and widened alveolar septae, with increased mucous in the airways that is consistent with chronic asthmatic bronchitis. Charcot-Leyden crystals were seen and are evidence of eosinophil degranulation. The small vessels of the lung had areas of muscular thickening without intimal proliferation, consistent with mild pulmonary hypertension. The initial bone marrow biopsy before death failed to engraft. A biopsy obtained during autopsy demonstrated trilineage hematopoiesis, but was hypercellular with a marked increase in the number of eosinophils. In all, the autopsy findings supported the diagnosis of HES.

\section{Role of Cardiac MRI}

Cardiac MRI was performed which included cine SSFP as well as GRE grid-tagging sequences to further evaluate myocardial function (Fig. 3). These sequences demonstrated severe myocardial hypokinesis in the mid to apical septum and the apex of the left ventricle, which was not clearly identifiable due to interference from the apical thrombus on transthoracic echocardiography. Post-contrast images performed 20 minutes following administration of gadolinium demonstrated a thin rim of abnormal hyperenhancement of the subendocardium throughout the left ventricle (Fig. 2). 


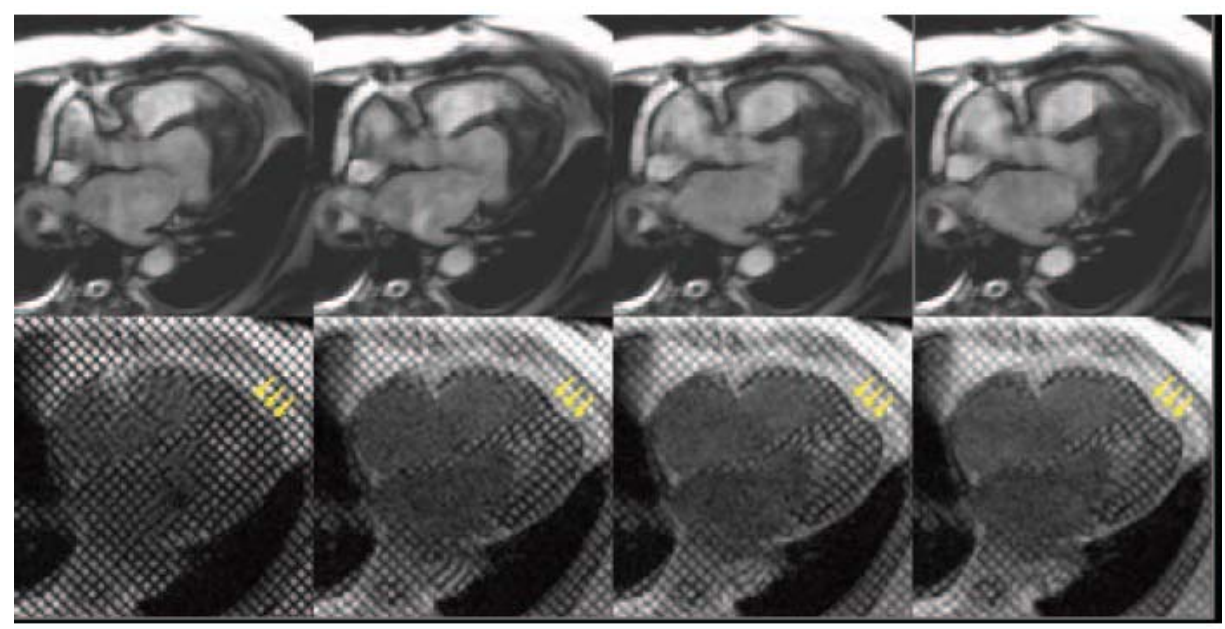

Fig. (3). Horizontal long-axis MRI images: cine steady-state free precession (top row) and GRE grid-tag images (bottom row) with both sequences arranged left (diastolic) to right (systolic). Grid tags clearly demonstrate poor contractile function (hypokinesis) in the apical region characterized by lack of deformation between diastole and systole (yellow arrows). This hypokinetic apical myocardium has normal signal characteristics on all other sequences (Fig. 2).

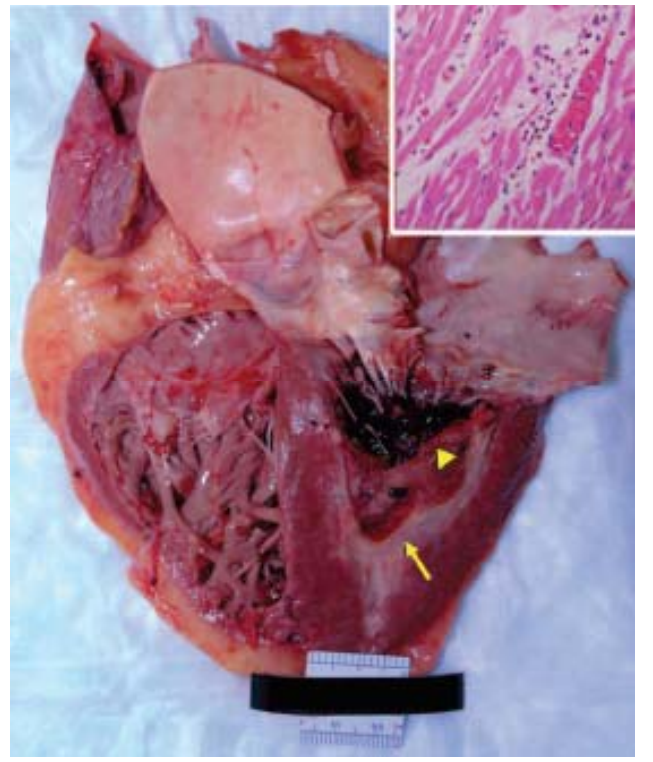

Fig. (4). A mural thrombus is in the left ventricle and involves the chordae tendinae of the mitral valve (arrowhead). The thrombus is overlying a fibrous, thickened endocardium (arrow). Insert - Scattered eosinophilic infiltration and fibrosis within the myocardium of the left ventricle (Hematoxylin \& Eosin stain, original magnification - 400x).

MRI also demonstrated a large intracavitary thrombus, which did correlate with findings on echocardiography (Figs. $1 \& 2)$.

Although cardiac MRI has been described previously in cases of eosinophilic myocardial infiltrative diseases, we believe this case is unique in demonstrating the direct pathologic correlation with the cardiac MRI findings. In particular, the rim of subendocardial hyperenhancement was readily apparent and correlates directly with fibrotic tissue on the pathologic specimen (Figs. 2 \& 4). Also, we believe this case is unique in documenting the myocardial systolic dysfunction on both SSFP and GRE grid tagging sequences, which may be subtle and underappreciated on transthoracic echocardiography. Most importantly, this case demonstrates that although the myocardium deep to the rim of subendocardial fibrosis had a normal enhancement pattern and was grossly normal on the pathologic specimen, it clearly possessed abnormal systolic function. In fact, the histologic assessment of this region demonstrated scattered areas of inflammation consisting predominantly of eosinophils and focal myocyte necrosis (Fig. 4). Based on these findings, it suggests that the inflammatory effects extend deeper into the myocardium than the area of subendocardial fibrosis alone.

\section{DISCUSSION}

Endomyocardial damage in HES begins with an acute necrotic stage. This initial stage appears to be caused by endomyocardial eosinophilic infiltration and degranulation which releases inflammatory mediators (Major Basic Protein, cytokines, leukotrienes, etc.) causing marked cytotoxic effects and a locally induced pro-thrombotic state. As the inflammatory cascade and hypereosinophilic state continues to propagate, the cytotoxic effects and pro-thrombotic state cause permanent endothelial and myocyte damage. This damage results in a chronic fibrotic stage characterized by contractile dysfunction, fibrosis, and infarction, leading to ventricular cavity obliteration and severe hemodynamic impairment [5]. Nearly always, the disease is complicated by ventricular thrombus formation. The bulk of the thrombus can, in and of itself, cause restrictive physiology. In patients with unexplained hypereosinophilia, testing of the peripheral blood and bone marrow is necessary to rule-out a myeloproliferative neoplasm, a primary eosinophil neoplasm, a lymphoid neoplasm, or a mast cell neoplasm [6]. In this case, a peripheral blood test for the $t(5: 12)$ platelet-derived growth factor receptor (PDGFR) $\beta$ fusion gene was obtained prior to death and it was negative, arguing against a primary eosinophilic leukemia [7, 8].

While some case reports have demonstrated the utility of cardiac MRI in HES, none to our knowledge have demonstrated a histopathologic correlation, which validates the utility of MRI in diagnosis and treatment of Loeffler's endocarditis [8-12]. This study also suggests that cardiac MRI can also provide guidance regarding the optimal anatomic site 
for biopsy [9]. In addition, cardiac MRI has the potential as a non-invasive method to evaluate and monitor the response of therapy for HES $[13,14]$. As demonstrated by this case report, cardiac MRI can detect subendocardial contractile dysfunction and fibrosis which can lead to a restrictive cardiomyopathy that is not easily detected by transthoracic echocardiography and may be useful in early diagnosis, when echocardiography may be too insensitive to detect subtle changes in ventricular structure and function. Future studies are necessary to determine the impact of early utilization of cardiac MRI in the diagnosis and management of HES patients and whether cardiac MRI could potentially obviate the need for cardiac biopsy.

\section{Financial Disclosure(s)}

K. Chad Hilty, MD - no conflicts of interest, James A. Koonce, MD - no conflicts of interest, Roger W. Stone, MD - no conflicts of interest, Luis Ramos-Duran, MD - no conflicts of interest, Ashley L. Pyle, MD - no conflicts of interest, Nicholas I. Batalis, MD - no conflicts of interest, Andrew W. Hardie, MD - no conflicts of interest, and Marian H. Taylor, MD - no conflicts of interest.

\section{Statement of Responsibility}

The authors had full access to the manuscript and take responsibility for its integrity. The authors have read and agree to the manuscript as written. This is an original manuscript and any data from previously published manuscripts were used with permission of the original author(s).

\section{REFERENCES}

[1] Spector HI. Loeffler's Syndrome (Transient Pulmonary Infiltrations with Eosinophilia). Chest 1945; 11: 380-91.
[2] Hardy WR, Anderson RE. The hypereosinophilic syndromes. Ann Intern Med 1968; 68(6): 1220-9.

[3] Parrillo JE, Borer JS, Henry WL, Wolff SM, Fauci AS. The cardiovascular manifestations of the hypereosinophilic syndrome. Prospective study of 26 patients, with review of the literature. Am J Med 1979; 67(4): 572-82.

[4] Fauci AS, Harley JB, Roberts WC, Ferrans VJ, Gralnick HR, Bjornson BH. NIH conference. The idiopathic hypereosinophilic syndrome. Clinical, pathophysiologic, and therapeutic considerations. Ann Intern Med 1982; 97(1): 78-92.

[5] Jaffe H, Stein, Vardiman. Tumors of Haematopoietic and Lymphoid Tissues: Lyon: International Agency for Research on Cancer 2008.

[6] Valent P. Pathogenesis, classification, and therapy of eosinophilia and eosinophil disorders. Blood Rev 2009; 23(4): 157-65.

[7] Gleich GJ, Frigas E, Loegering DA, Wassom DL, Steinmuller D. Cytotoxic properties of the eosinophil major basic protein. J Immunol 1979; 123(6): 2925-7.

[8] Kleinfeldt T, Ince H, Nienaber CA. Hypereosinophilic Syndrome: A rare case of Loeffler's endocarditis documented in cardiac MRI Int J Cardiol 2009; [Epub ahead of print].

[9] Salanitri GC. Endomyocardial fibrosis and intracardiac thrombus occurring in idiopathic hypereosinophilic syndrome. Am J Roentgenol 2005; 184(5): 1432-3.

[10] Bishop GG, Bergin JD, Kramer CM. Hypereosinophilic syndrome and restrictive cardiomyopathy due to apical thrombi. Circulation 2001; 104(2): E3-4.

[11] Syed IS, Martinez MW, Feng DL, Glockner JF. Cardiac magnetic resonance imaging of eosinophilic endomyocardial disease. Int J Cardiol 2008;126(3): e50-2.

[12] Puvaneswary M, Joshua F, Ratnarajah S. Idiopathic hypereosinophilic syndrome: magnetic resonance imaging findings in endomyocardial fibrosis. Australas Radiol 2001; 45(4): 524-7.

[13] Baccouche H, Yilmaz A, Alscher D, Klingel K, Val-Bernal JF, Mahrholdt H. Images in cardiovascular medicine. Magnetic resonance assessment and therapy monitoring of cardiac involvement in Churg-Strauss syndrome. Circulation 2008; 117(13): 1745-9.

[14] Chandra M, Pettigrew RI, Eley JW, Oshinski JN, Guyton RA. Cine-MRI-aided endomyocardectomy in idiopathic hypereosinophilic syndrome. Ann Thorac Surg 1996; 62(6): 1856-8. 